\title{
Autogenous tooth transplantation an alternative to implant ??? - a review
}

Dr. Vijaykumar J Girhe PG Student

Dr. Rajay Kamath Asst.Professor

Dr. David P Tauro Professor \& Head

Department of Cranio-Maxillofacial, Plastic and Reconstructive Surgery

\section{INDICATIONS}

While there are many reasons for autotransplanting teeth, tooth loss as a result of dental caries is the most common indication, especially when mandibular first molars are involved. First molars erupt early and are often heavily restored. Autotransplantation in this situation involves the removal of a third molar which may then be transferred to the site of an unrestorable first molar.Other conditions in which transplantation can be considered include tooth agenesis (especially of premolars and lateral incisors), traumatic tooth loss, atopic eruption of canines, root resorption, large endodontic lesions, cervical root fractures, localized juvenile periodontitis as well as other pathologies. Successful transplantation depends on specific requirements of the patient, the donor tooth, and the recipient site.

\section{CANDIDATE CRITERIA}

Patient selection is very important for the success of autotransplantation. Candidates must be in good health, able to follow post-operative instructions, and available for follow-up visits. They should also demonstrate an acceptable level of oral hygiene and be amenable to regular dental care. Most importantly, the patients must have a suitable recipient site and donor tooth. Patient cooperation and comprehension are extremely important to ensure predictable results.

RECIPIENT SITE CRITERIA

The most important criteria for success involving the recipient site is adequacy of bone support. There must be sufficient alveolar bone support in all dimensions with adequate attached keratinized tissue to allow for stabilization of the transplanted tooth. In addition, the recipient site should be free from acute infection and chronic inflammation.

DONOR TOOTH CRITERIA

The donor tooth should be positioned such that extraction will be as atraumatic as possible. Abnormal root morphology, which makes tooth removal exceedingly difficult and may involve tooth sectioning, is contraindicated for this surgery. 1 Teeth with either open or closed apices may be donors; however, the most predictable results are obtained with teeth having between one-half to two-thirds completed root development.1-5 Surgical manipulation of teeth with less than one-half root formation may be too traumatic and could compromise further root development, stunting maturation or altering morphology. When root development is greater than two-thirds, the increased length may cause encroachment on vital structures such as the maxillary sinus or the inferior alveolar nerve. Furthermore, a tooth with complete or near complete root formation will generally require root canal therapy, while a tooth with an open apex will remain vital and should continue root development after transplantation. In the latter case, successful transplantation without the need for further endodontic therapy is usually seen.

\section{CONCLUSION}

Although autotransplantation has not been established as a traditional means of replacing a missing tooth, the procedure warrants more consideration. Recent studies clearly demonstrate that autotransplantation of teeth is as successful as endosseous dental implant placement. Minimum acceptable success rates for endosseous titanium dental implants are $85 \%$ after 5 years and $80 \%$ after 10 years. 21 For younger patients, autotransplantation may also be considered as a temporary measure. The transplant can replace missing teeth to ensure preservation of bone until growth has ceased and then, if necessary, the patient can become a candidate for implants. With appropriate patient selection, and presence of a suitable donor tooth and recipient site, autogenous transplantation should be considered as a viable option for treatment of an edentulous space. 